\title{
CEN activities on enterprise modelling and enterprise model execution and integration services
}

\author{
D.N. Shorter
}

Convenor of CEN TC310 WG1

IT Focus, Folia, Flowers Hill, Pangbourne, Berks RG8 7BD, UK

Telephone: 01734 843949, Fax: 01734 842493,

Email: david@itfocus.demon.co.uk

\begin{abstract}
Today's manufacturing enterprise needs both increased integration of its processes and resources, while at the same time retaining the ability to respond rapidly to an ever changing market requirements. Enterprise modelling aims to meet these potentially conflicting objectives. The paper describes CEN's work to establish the standards that are needed to support Enterprise Modelling for manufacturing, in particular on a framework of concepts, an evaluation of and preliminary standards for modelling constructs, and a statement of requirements for the environment in which model constructs are to be developed and executed (EMEIS).
\end{abstract}

\section{OBJECTIVES FOR THE WORK}

There are two potentially conflicting business drivers which are of particular importance for today's manufacturing enterprise. These are:

- Developing, maintaining and improving the integration of manufacturing processes and resources.

Redesigning manufacturing and business processes to meet changing market conditions.

Integration of data and integration of communications are prerequisites and other standards work is addressing these requirements. This paper describes a third and complementary approach - integration through an enterprise model. 
Management techniques such as Just in Time, Business Process Re-engineering, the Virtual Enterprise etc. all place new demands on the ability to redeploy manufacturing resources flexibly, and to do this in such a way as to retain the degree of integration necessary to reduce work in progress and associated lead times. Enterprise modelling is intended to be both to be supportive of manufacturing integration, and to facilitate organisational and process redesign.

\section{THE ENTERPRISE MODELLING APPROACH}

Enterprise Modelling for manufacturing is a technique which sets out to meet these objectives by explicitly modelling a manufacturing enterprise.

For a particular enterprise, the model needs to represent all relevant aspects of the agents, processes and resources which are involved; to do this in a way which ensures consistency and completeness; and to provide the possibility of executing these models to direct enterprise operations (possibly after a translation process).

CEN TC310 WG1 (previously CEN/CENELEC WG/ARC) is a standardization committee which has been working to develop the standards necessary for such models in five work areas:

- Producing a Framework for Enterprise Modelling which sets out the general requirements and terminology for such enterprise models.

- Evaluating technologies which might form general components or 'building blocks' for models.

- Developing a Statement of Requirements for the IT services (EMEIS) that are required for the execution and integration of model components, following an evaluation of existing approaches.

- Producing a vocabulary for CIM systems architecture for guidance to those working in the field, and as an organising document for its own work.

- Working to produce a PreStandard for building blocks, known as 'Constructs for Views'. This is the main item of on-going work.

How the WG is organised will be discussed after describing these work areas in somewhat more detail.

\section{THE ENV 40003}

In 1991 CEN published the Framework for Enterprise Models (ENV 40 003) which defines '... a framework for ... computer-based modelling of enterprises, focusing on Discrete Parts Manufacturing. Models generated using this framework will ultimately be computer executable and possibly enable the daily operations of an enterprise to be run, monitored and controlled by such models.' This Framework was developed from a substantial contribution from the AMICE consortium which produced the CIMOSA concept (Open System Architecture for CIM), with further inputs from industry and academia.

In particular, the modelling concepts of the Framework support:

- Genericity - the ability to specialise from general models to partial models used by a specific industry sector, and even by a particular enterprise.

- Model lifecycle issues, e.g. the ability to encompass the three main phases of requirements specification, design and implementation 
- The selective perception provided by Views. Each View concentrates on some aspect of the model for a particular purpose and hide irrelevant aspects to reduce complexity. Whether there is to be just one set of views is still a matter of some discussion, but the ENV (and the current work of the WG1) has adopted for the moment the four views of Function, Information, Resource and Organisation.

The first two of these Views, Function and Information, correspond to 'classical' functional and information analysis. The third, Resource, supports the modelling of the use, consumption and production of (largely) material resources. The last, Organisation, is more novel - it allows for the specification of which part of the business has the responsibility for initiating which manufacturing activity and under what conditions. (It is somewhat similar to the use of explicit modelling of workflow in office environments.)

The explicit representation of enterprise entities according to the Views of the ENV provides the following benefits:

- Function View: Improved flexibility and ability to adapt through more flexible coupling between processes.

- Information View: Capture of 'organisational memory' and improved information re-use; a prerequisite for inter-enterprise trading, and for a manageable and robust IT structure.

- Resource View: Again flexibility and adaptability; reduction of work in progress.

- Organisation View: Increased ability to adapt and respond to changing market conditions and appropriate manufacturing logistics.

These Framework concepts have, of course, a close correspondence to the modelling framework of CIMOSA on which they were based.

How the model is implemented, e.g. as computer-interpretable data, is not the main issue for WG1. For example, while object-oriented approaches are very powerful in terms of their ability to handle complexity, they are not the only approach to implementation. All object-oriented methods contain some key technical concepts which can help in managing the representation of complex requirements and designs (encapsulation, inheritance, polymorphism). But until recently, the tools which allow an $\mathrm{OO}$ analysis of a manufacturing situation and the design of appropriate software structures did not easily lead to the development of executable code. Generally at some stage the developer is required to develop fragments of code to supply the required functionality. This situation is changing however as new tools become available.

\section{EVALUATION REPORT ON CONSTRUCTS}

After the development of the ENV 40003 , WG1 set out to clarify requirements for generic model components (components which could be specialised to meet particular enterprise needs). These components needed to be in accord with the ENV, and also capable of computer representation and checking.

A number of detailed requirements were identified from ENV 40003 . These were then consolidated into a set of detailed questions that should be asked about particular modelling methods.

Following a call for input, the WG received 11 sets of material to review. Each was summarised 
in terms of its source, documentation available, status, description, evaluation summary and the implications for the WG in its task of producing standardized constructs. Tables contained the detailed evaluations for each method.

At the time that the evaluation was carried out (1992), the main conclusions were that:

The different methods covered to a varying extent some part of what was needed - but no one method was sufficiently comprehensive for the WG's objectives.

- The more comprehensive approaches were research projects that had not yet been sufficiently demonstrated to be practicable.

- Generic model components could be realised using both function oriented and object oriented approaches and that any standard needed to be open to both.

- Generic model components particularly designed to address one View of the ENV 40003 could not be studied in isolation from components designed for the needs of other Views.

This report was published by CEN as R-IT-06 in April 1993, and has been republished in late '94 in a special issue of 'Computers in Industry' on CIM Architectures (Ed: Prof. H Wortmann, Sept. 1994, Elsevier Science B.V.) by kind permission of the Nederlands Normalisatie-instituut.

\section{REQUIREMENTS FOR ENTERPRISE MODEL EXECUTION AND INTEGRATION SERVICES (EMEIS)}

\subsection{Background}

The work item 'Enterprise Model Execution and Integration Services' is aiming to provide a specification of the standards required for the (possibly distributed) computing environment that is needed to execute an enterprise model. WG1 has now produced a statement of requirements which is outlined in this section and as assessment of available technology described in Section 7. This statement will be developed into another ENV after comments and further inputs have been received from interested parties.

\subsection{The 'basic reference model' for EMEIS}

In order to put the different kinds of requirement in an overall context, the WG developed a simple model of the key concepts and their relationships. This had three main components:

- Model development services - the collection of services which in total provide the environment within which model components are developed and tested before release to the EMEIS for use as an executable model of an enterprise, or as part of such a model.

- Model execution services - the collection of services which (i) embed a model component into EMEIS, so converting it into a runnable entity and (ii) provide all the operations services that are particular to the execution of such runnable entities, or for the provision of other CIM-specific services, over and above the IT Base services .

- IT Base services - the general IT services which are not CIM specific. These are concerned with systems qualities such as portability of applications, interworking between open systems and distribution transparency in distributed platforms. 
The relationships between these are illustrated in Figure 1.

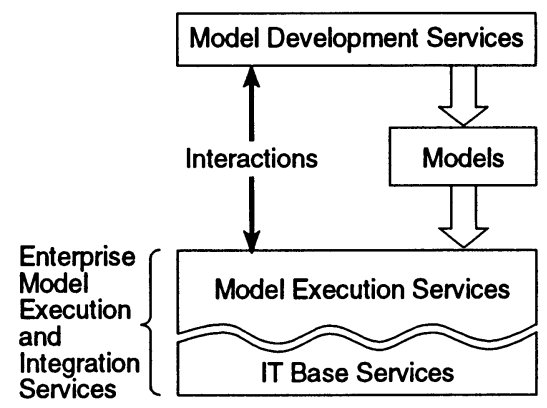

Figure 1 The reference model for EMEIS

\subsection{Guiding principles for EMEIS requirements}

In developing the statement of requirements, the WG articulated and used a number of guiding principles. These were:

- Conformance to existing standardization, including ENV 40003.

- Openness to different modelling approaches - because enterprise models 'compliant' with ENV 40003 are difficult to find.

- Description of EMEIS in, at least, two levels of abstraction (functional representation and IT representation).

The use of services and service requesters (clients) to achieve a degree of technological independence.

- Services to be encapsulated (describe the 'what' not the 'how').

- System structuring according to system theory.

- Use of an Applications Program Interface to provide transparency of service provision, application portability etc.

- Use of protocols to control data exchange.

- EMEIS as a requirements framework should contain abstractions for the mechanisms which support the EMEIS requirements, not the mechanisms themselves.

The principle embedded in ENV 40003 of enterprise integration through the use of an explicit model led the WG to make some particular assumptions about the relationships between:

The environment and services which are needed to develop the model or model component (MDS),

- The services required to embed and operate the model (MXS),

- The General IT Services which provide access to enterprise data. 


\subsection{CIM-specific requirements}

Led by consideration of particular requirements and design rules for CIM, including that of the essentially physical nature of the manufacturing process, the following requirements were identified (not all of which are unique to CIM but which have been given a particular interpretation):

- What is to be provided by MDS, including the necessary scope of an enterprise model and the stages in its development.

- The required granularity of model, including guidance on when to stop modelling.

The need to support three different types of model component (compiled, interpretable and parameter-driven).

- The ability to compose models, possibly expressed in different languages corresponding to different Views, into one integrated model; the need for a mechanism to support that process; and the need to address life-cycle issues for model components.

- What is involved in embedding a model to make it an executable entity.

- Where model bindings should be established, corresponding to the rate of change (from organisational change to resource management to immediate 'make' instructions).

- The services that are needed to provide the resources necessary for a model component to execute.

- The need for binding mechanisms in both MXS and MDS (both early and late binding).

- The characteristics of the information that needs to pass between the MDS and MXS.

- Incremental development of model components.

- End user visibility of model components and the operation of those components (or assemblages of these).

Based on these requirements, and especially the principle of services, the report proposes a way of identifying what future EMEIS standards might be needed. An initial set of possibilities is identified as:

Acommon mechanism for describing model components in different languages and provided by different suppliers.

- A common mechanism for interactions (messages) between MDS and MXS.

- A standardized way of describing model behaviour.

- A common set of semantics for the states of model components and the signalling of those states.

A common procedure or process for declaring, registering and withdrawing model components.

It should be noted that there are many similarities here with the requirements for Integrated CASE tools in software engineering.

\subsection{General requirements for EMEIS}

Services are classified into three groups (although the boundary between EMEIS and IT Services is not clear cut): 
Model Development Services, MDS

Model Execution Services, MXS, in turn comprising

- Model Embedding Services

- Model Operation Services

- General IT Services

Often services can be decomposed into other services, but from the point of view of specifying EMEIS requirements, decomposition is required only where it is desired to constrain EMEIS services and service components in some way.

The report sets out a list of IT-related service requirements for MDS and MXS, covering such issues as lifecycle requirements, simultaneous development, namespace management, recovery procedures, etc.

General IT services have to provide support for model communication with the real world, including access to the current state of the enterprise, the presentation of information to human operators and the acceptance of human intervention etc. These services are to provided using possibly heterogeneous and distributed IT resources. General IT services should be decoupled as far as possible from the underlying technology, make maximum use of services which are the subject of available standards, and use existing services and protocols for agent protocols. Again, a list contains more detailed requirements.

\section{EVALUATION OF EMEIS CONTRIBUTIONS}

In preparing the review of relevant project initiatives, CEN TC310/WG1 prepared a checklist against which the contributions were reviewed. Experience in this evaluation contributed to the Statement of Requirements described above. A necessarily condensed version of the checklist follows:

Q1 What visibility is there of concepts of model, models, model components?

Q2 How does the model represent derived functionalities? Embedded processes (include control, timing and behaviour)? Necessary information and other resources? What else is represented explicitly in this approach (e.g. obligations as in Eiffel)?

Q3 What is the level of integration, from (lowest) communication between running model processes, through shared data or services, to meta-models, shared semantics and semantic unification (highest)?

Q4 What binding paradigm is used (concept, interpretation)? Where does binding take place etc.?

Q5 What modelling language is used to represent the model(s) or model components and what attributes of the model does this language capture?

Q6 Does the model support predictability of run-time behaviour (including performance)? Is the use of resources and methods predetermined or opportunistic?

Q7 What is the paradigm used for invocation of executable model component? (Eager evaluation, lazy evaluation, controlled evaluation, context-driven application protocol)?

Q8 What CIM-specific semantics or application-specific semantics are visible during model execution? (e.g. CIM ontology, class library or CIM-specific notions such as consumable resources) 
Q9 Is the question of lifecycle (of model(s), of model component) addressed and, if so, how? Is the approach used linked to the binding mechanism used, e.g. by maintenance of a binding trail allowing components to be withdrawn or replaces and the consequences managed?

Q10 What is the definition of service in the contribution being evaluated? The ISO TC184 SC5 WGl's definition was used as a reference concept here.

Q11 How are the 'General IT Services' and other services accessed? Five sub questions asked about: the assumed execution environment; properties of the execution environment; visibility of IT service components; when and how such services are invoked; and how Enterprise Model Execution and Integration Services are characterised in the approach.

Q12 How is the 'Level' between the model and its executing environment (the one in which the model is executed, its execution environment) described?

These questions were used as a set of concerns to assess six contributions, as summarised in Table 1. Each proposal was summarised based on the contributions supplied. The WG wishes to express its thanks to the sources of these contributions, and also for the assistance giving in reviewing the assessments and clarifying various points.

Table 1 Contributors to the EMEIS evaluation

CIMOSA N198, N199, N245 [N198 (CIMOSA, Group A) 'Framework for Integrating Infrastructure', CIMOSA, 15 June 1992; N199 (CIMOSA, Group A) 'Formal Reference Base for IIS', CIMOSA, 15 June 1993; N245 (CIMOSA, Group A) 'Relation between CIMOSA model and IIS, Kurt Kosanke, 11/5/93; pages 2-3 (N245).

Flatau N219 'Framework for an Integrating Infrastructure' Ulrich Flatau and Dieter Wüsterfeld, October 1992. This proposal is essentially that contained in ISO TC184 SC5 WG1's N300 as of late 1992.

MIDA 246, MIDA: An Open Systems Architecture for the Model-Oriented Integration of Data and Algorithms; M. H. R. Michalski, D. Solte, F. Vicuna; FAW Ulm, 11/ 5/93; pages $1 . .25$.

PISA N238, Framework for IIS, W Gielingh, 5/3/93.

TOVE N239, A common-sense model of the enterprise, Mark S Fox, J Chionglo, Fadi G Fadel, 19/2/93.

ISA A framework for information systems architecture, J A Zachman, IBM Systems Journal, Vol 26, No 3, (C) 1987 IBM; Extending and formalising the framework for information systems architecture, J F Sowa and J A Zachman, IBM Systems Journal, Vol 31, No 3, (C) 1992 IBM.

Following the summary of each project, an assessment was made using the questions above as a common frame of reference. These assessments were later sent to the projects and revised where necessary after clarification.

The main conclusions of the evaluation report were that: 
- Carrying out the assessments made a major contribution to WG1's understanding of requirements, and greatly contributed to the Statement of Requirements described earlier. It would have been desirable to align the questions above with that Statement, and then to repeat the assessment exercise but this was not possible in the time available.

- Several of the initiatives contain requirements statements and design proposals covering at least some part of EMEIS requirements - but none encompasses all the reference concepts or functionality seen as necessary by WG1. At the time of the evaluation (mid-late 1993) most (all?) of the contributions were research or development projects, and industrial experience was lacking, especially for MXS.

\section{FUTURE WORK ON EMEIS}

The reports on EMEIS Statement of Requirements and the EMEIS evaluations are being published by CEN to elicit wider feedback. It is then likely that the work on EMEIS requirements will be reworked and submitted for ballot as a European PreStandard (ENV) on EMEIS Requirements.

At a suitable time, probably as the work on Constructs for Views nears completion, the WG intends to issue a call for new input, based on a somewhat revised Statement of EMEIS Requirements, or against an initial draft of the intended ENV.

During these developments, the WG is particularly concerned to maintain appropriate liaisons and further develop these where necessary, to ensure that new standards are developed only where the requirements cannot be fulfilled by existing or developing standards. Liaisons with ISO TC184 SC5 WG1 and WG4 will continue through cross membership and exchange of documents. Other liaisons that have been identified as important are with the IFAC/IFIP Task Force on Architectures for Enterprise Integration, and with a number of activities in ISO/IEC JTC1 SC21.

At the risk of introducing unmanageable complexity, the WG would welcome expressions of interest in liaison from other standardization groups. Section 11 describes how experts wishing to contribute more directly can be nominated to participate more directly in the work of WG1.

\section{VOCABULARY FOR CIM SYSTEMS ARCHITECTURE}

During the course of its work, WG1 identified the need for 'common and documented understanding of terms and definitions in the field of CIM Systems Architecture.' This is particularly a problem because many of the words used are already used with somewhat different meanings in other fields, e.g. in Systems Engineering, and in IT generally.

The terms and definitions of the vocabulary were produced taking into account already established terms in ISO (in particular the terms used by ISO TC184 SC5 WG1) and elsewhere. The group is maintaining links with others active in this field and is exchanging documents.

The draft N26 was been produced in 2/3/94 and recommended for circulation by CEN. Depending on that response, the document may progress to a standards document. Alternatively it could remain as a very necessary aid to WG1's future work. 


\section{PRESTANDARD ON CONSTRUCTS FOR VIEWS - WORK IN PROGRESS}

WG1 is now working on the definition and representation of the further concepts that are required. In particular, if model components are to be procured from multiple sources, or indeed later reused elsewhere within an enterprise, then there needs to be an adequate way of describing the significant properties of a model in terms of these concepts (a standardized modelling language), and of specifying precisely what each concept signifies (e.g., in a formal specification in a language such as EXPRESS).

A further desirable objective is for a common graphical representation to allow easier understanding of model components and the relationships between these - for both model-user and model-developer.

This work item is setting out to meet these objectives by creating a European Standard (initially an ENV) as an input to and a precursor of international work in ISO. Substantial input has been received from two European initiatives and WG1 also maintains links with international developments. However, the work is not just standardizing the status quo - it is open to new proposals for constructs in accordance with ENV 40003.

\section{CEN TC310 WG1, SYSTEMS ARCHITECTURE FOR MANUFACTURING}

CEN TC310 WG1 is a working group of CEN, the European Committee for Standardization located in Brussels, and in particular of Technical Committee 310, Advanced Manufacturing Technologies. The mission of TC 310 is to make sure that the standards required for Advanced Manufacturing Technologies are available to and known by European industry.

Priority is given to using international standards wherever this is possible and to undertake work in Europe only where a need has been identified which is not being met by developments internationally. For example, CEN TC310 WG1 maintains an active liaison with ISO TC184 SC5 WG1, Modelling and Architecture, and several members are active participants in both committees. The work programme and strategy of TC310 is documented in a CEN/CENELEC document known as M-IT-04, which provides an organised classification of work items, their time scales and the strategy for their development..

The current work of TC 310 can be grouped into four main areas:

Product Data Exchange, concentrating on the specific European needs for STEP developments,

- CIM Systems Architecture, the subject of this overview,

- Standards Parts Libraries - standards for representation of standard parts to be used by CAE systems,

- Ergonomics, where future directions for Europe are being set by a workshop,

The principal objective for WG1, CIM Systems Architecture, is to ensure that the requirements of European industry are met, so that maximum advantage can be taken of standardization for enterprise modelling and the use of development environments that will influence the industrial organisation, management and manufacturing approach to improving efficiency. WG1 has noted that enterprise modelling is potentially a powerful enabler in planning and implementing organisational and structural change. 
A secondary objective is to ensure that existing expertise is deployed in the preparation of the standards, and that the necessary information is available to interested parties in the form of publicly available standards.

Enterprise integration via enterprise modelling is a new technology and requires R\&D input for progress to be made. This standards work therefore has a strong dependence on the results of European research initiatives such as ESPRIT, and is acting as an important technology transfer mechanism in making those results available to European industry.

\section{WHERE NEXT}

Having completed a Framework for Enterprise Modelling as ENV 40003, WG1 is now working to standardise requirements for constructs in accordance with that framework, and to standardize requirements for the environment within which models will be executed. When complete, this work will form the basis for the longer term development of standardized constructs and services within the executing environment.

As constructs become standardized, they will be more widely accepted and be able to deliver similar benefits to a wider community of users. At the same time, greater acceptance of these concepts will encourage further tool development, the establishment of libraries of useful particular models and increased availability of skilled expertise to apply and teach the technology.

Standardization offers particular benefits in promoting exchangeability and re-use of model components. It should also encourage the development of model-related products and services by providing a recognised and stable basis for these developments (e.g., a common and nonproprietary form of representation, common usage of terms etc.).

By participating in this work, companies, research organisations and consultants can influence its direction and gain insights into how these developments can be applied in their own business, products or services. In particular, participation can allow the merits of a particular technical approach to be presented to an informed group of experts, and if accepted, to become a constituent part of an emerging European standard. (Participation in the work is open to experts nominated by their National Standards Organisation, who must be members of CEN, and occasionally to invited experts.) 


\section{BIOGRAPHY}

David Shorter is an independent IT consultant trading as IT Focus, offering strategic consultancy in IT-related matters based on his experience as Director of IKBS for the UK Alvey programme, as manager of the external research and technical coordination programmes for SD-Scicon and on his work in the development of Frameworks and Reference Models for enterprise modelling, and the representation of these using object-oriented techniques. He is also convenor of the CEN Working Group on Enterprise Modelling for CIM (TC310 WG1). 